\title{
Contrast-enhanced ultrasound in the diagnosis of hepatocellular carcinoma ${ }^{\text {ti }}$
}

\author{
Riccardo Lencioni ${ }^{1, *}$, Fabio Piscaglia ${ }^{2}$, Luigi Bolondi ${ }^{2}$ \\ ${ }^{1}$ Division of Diagnostic and Interventional Radiology, Department of Oncology, Transplants, and Advanced Technologies in Medicine, \\ University of Pisa, Pisa, Italy \\ ${ }^{2}$ Division of Internal Medicine, Department of Internal Medicine and Gastroenterology, University of Bologna, Bologna, Italy
}

\begin{abstract}
Surveillance of patients at risk of developing hepatocellular carcinoma (HCC) is based on ultrasound (US) examinations performed at either 6 or 12 month intervals. Early detection of HCC in patients with cirrhosis is a challenging issue, since the different entities that are involved in the multi-step process of hepatocarcinogenesis - such as low-grade and high-grade dysplastic nodule - share common US features. Contrast-enhanced US allows reliable detection of arterial neoangiogenesis associated with a malignant transformation. Several reports have shown that the ability of contrast-enhanced US to diagnose HCC currently approaches that of optimized multidetector computed tomography (CT) or dynamic magnetic resonance (MR) imaging protocols. The use of contrast-enhanced US to characterize nodular lesions in cirrhosis has been recently recommended by the clinical practice guidelines issued by the European Federation of Societies for Ultrasound in Medicine and Biology and the American Association for the Study of Liver Diseases. However, contrast-enhanced US has not resulted in any significant improvement in the ability of US to detect small tumor foci, since a comprehensive assessment of the whole liver parenchyma cannot be accomplished during the short duration of the arterial phase. Hence, CT or MR imaging are still mandatory for proper intrahepatic staging of the disease.
\end{abstract}

(c) 2008 European Association for the Study of the Liver. Published by Elsevier B.V. All rights reserved.

Keywords: Cirrhosis; Hepatocellular carcinoma; Ultrasound; Contrast agent; Computed tomography; Magnetic resonance imaging

\section{Introduction}

Despite advances in computed tomography (CT) and magnetic resonance (MR) imaging, ultrasound (US)

\footnotetext{
Associate Editor: M. Colombo

The authors declare that they do not have anything to disclose regarding funding from industries or conflict of interest with respect to the manuscript.

* Corresponding author. Address: Division of Diagnostic and Interventional Radiology, Cisanello University Hospital, Via Paradisa 2, IT-56124 Pisa, Italy. Tel.: +39050 992509; fax: +39050 551461.

E-mail address: lencioni@med.unipi.it (R. Lencioni).

Abbreviations: AASLD, American Association for the Study of Liver Diseases; CT, computed tomography; DN, dysplastic nodule; EASL, European Association for the Study of the Liver; HCC, hepatocellular carcinoma; MR, magnetic resonance; US, ultrasound.
}

continues to play a key role in the diagnostic algorithm of hepatocellular carcinoma (HCC). According to the European Association for the Study of the Liver (EASL) and the American Association for the Study of Liver Diseases (AASLD), US is the recommended tool for surveillance of patients at risk of developing HCC $[1,2]$. Moreover, US is the imaging technique most commonly used worldwide to guide biopsy on suspicious nodules $[3,4]$. The introduction of US contrast agents and the development of contrast-specific scanning techniques have substantially increased the sensitivity and specificity of US in the detection and characterization focal liver lesions [5]. Recently, the European Federation of Societies for Ultrasound in Medicine and Biology has issued recommendations for the use of contrast agents in liver US, and the AASLD clinical 
practice guideline too has included contrast-enhanced US - along with contrast-enhanced CT and contrastenhanced MR imaging - among the techniques that can be used for non-invasive diagnosis of HCC in cirrhosis $[2,6]$. In this article, we review basic principles and techniques of contrast-enhanced US of the liver and we discuss advantages and limitations of contrastenhanced US with respect to the other imaging modalities in the HCC setting.

\section{Basic principles}

US contrast agents are stabilized microbubbles containing either air (first-generation contrast agents) or other gases (second-generation contrast agents). Contrast-specific techniques are based on the complex interactions between microbubbles and US beam. The major determinant of this interaction is the peak negative pressure of the transmitted US pulse, grossly reflected in the mechanical index. By scanning with a low peak pressure, microbubbles behave as linear backscatterers, alternatively contracting and expanding according to the positive and negative pressures of the sinusoidal sound waves. This results in a simple reflection of US echoes. As the incident peak pressure increases, microbubbles show non-linear characteristics, and start emission of harmonics. Harmonics are created since on the negative portion of the sound waves, microbubbles can become quite large, but on the positive portion there is a limit at which they can be compressed: this asymmetry is what constitutes the harmonic emission. With further increase in the peak pressure of the incident US field, however, the shell of the microbubbles is disrupted [5].

Contrast-specific techniques suppress linear US signals coming from tissues and use the non-linear response of microbubbles to enhance signals from the contrast agent over the background. The advent of second-generation agents has been instrumental in improving the ease and the reproducibility of the examination, since low solubility gases offer improved stability and more favourable resonance behaviour than air at low acoustic pressure [7]. Hence, contrast-specific imaging can be performed at a low mechanical index, thus preventing microbubbles disruption and enabling visualization of the dynamic enhancement pattern in real time over several minutes.

Unlike iodinated contrast agents for CT and gadolinium chelates for MR imaging, US contrast agents are not cleared by the kidneys. Therefore, it is not necessary to perform any laboratory test of renal function before administering them. In general, US contrast agents are very safe with a low incidence of side-effects. Serious adverse events in abdominal applications have been reported with a rate of $0.0086 \%$ [8].

\section{Technique}

A full assessment of the liver with baseline US is mandatory before i.v. injection of the contrast. Then, contrast-specific imaging mode at low mechanical index is selected. This will ensure adequate cancellation of tissue signals, with only major vascular structures and some anatomical landmarks such as the diaphragm remaining barely visible. In some equipment, simultaneous display of tissue and contrast signals has been implemented to ensure that a lesion seen on contrast-enhanced imaging can be concurrently detailed on conventional B-mode. The US contrast agent is administered intravenously via bolus injection followed by saline flush. Because of the dynamic nature of real time contrast-enhanced US, the investigation should be documented on video or digital media, storing clips for each vascular phase [6]. Complete examination of the liver using various sweeps is possible within a time frame of approximately 4-5 min.

Enhancement patterns at contrast-enhanced US are typically described during subsequent vascular phases (arterial phase, portal venous phase, and late phase), similar to contrast-enhanced CT or contrast-enhanced MR imaging (Table 1). An inherent advantage of US is the possibility to assess the contrast enhancement patterns in real time, with a substantially higher temporal resolution than $\mathrm{CT}$ or MR imaging and avoiding the need to predefine scan time points or to perform bolus-tracking. US contrast agents, however, have different pharmacokinetics with respect to iodinated contrast agents for CT and gadolinium chelates for MR imaging. US microbubbles are confined to the intravascular space, whereas the majority of currently approved contrast agents for $\mathrm{CT}$ and MR imaging are rapidly cleared from the blood pool into the extracellular space [9]. Hence, despite many similarities, the enhancement pattern of a given lesion at contrast-enhanced US may not be identical to that observed at CT or MR imaging (Fig. 1).

\section{Detection of HCC}

Surveillance programs aimed at early detection of $\mathrm{HCC}$ in patients at risk are based on US examinations

Table 1

Phases of contrast-enhancement in liver US imaging

\begin{tabular}{lll}
\hline Phase & $\operatorname{Start}^{\mathrm{a}}(\mathrm{s})$ & $\mathrm{End}^{\mathrm{a}}(\mathrm{s})$ \\
\hline Arterial phase & $10-20$ & $20-35$ \\
Portal venous phase & $30-45$ & $120-180$ \\
Late phase & $120-180$ & $240-360^{\mathrm{b}}$ \\
\hline
\end{tabular}

The individual global haemodynamic situation will influence the time of onset of the three vascular phases.

a Time of visualization from start of contrast injection.

b Until microbubble disappearance. 
a

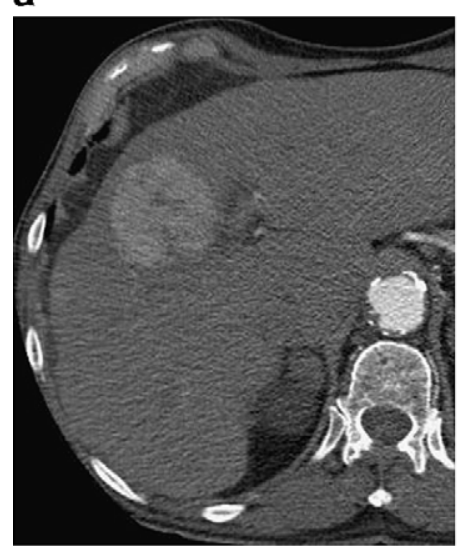

b

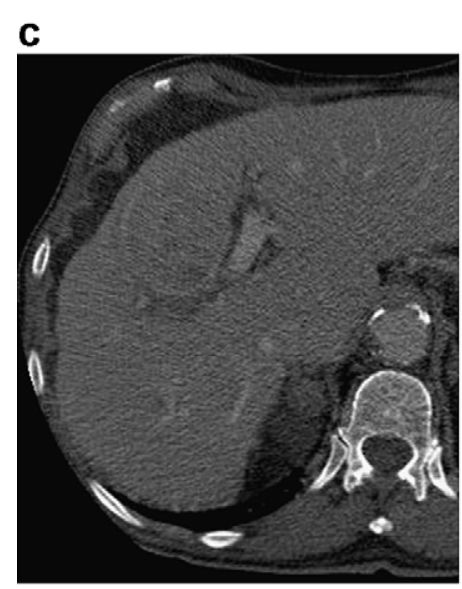

e

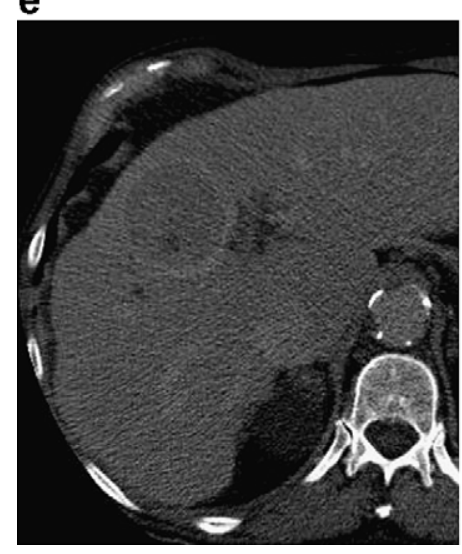

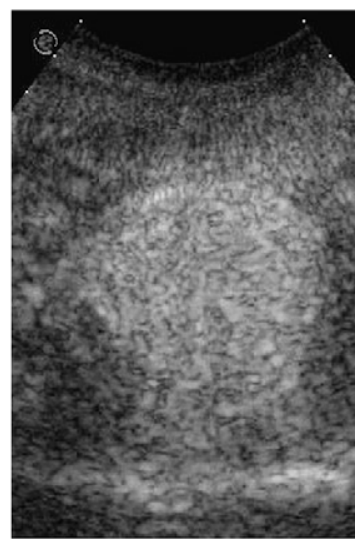

d

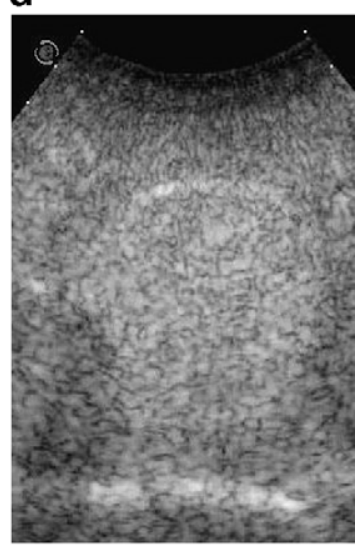

f

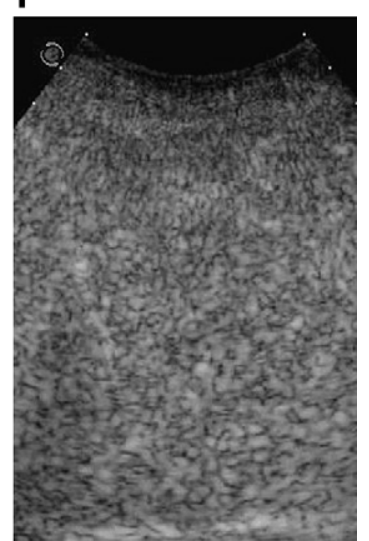

Fig. 1. Enhancement patterns of HCC at contrast-enhanced US and contrast-enhanced CT. The lesion shows clear-cut enhancement in the arterial phase both in the contrast-enhanced CT (a) and the contrastenhanced US study (b). However, contrast wash-out in the portal venous phase is better detected on CT (c) than on US (d). In the late phase, the tumor is hypoenhanced both on CT (e) and on US (f).

performed at either 6 or 12 month intervals $[1,2,10,11]$. Early detection of HCC, especially in patients with cirrhosis, is a challenging issue. Cirrhosis is characterized by fibrous septa and regenerative nodules. These features produce a coarse pattern on US, that may impair

identification of small tumors. Moreover, a comprehensive assessment of the liver parenchyma may sometimes be hampered by the patient's body habitus, colonic interposition, or morphologic changes induced by cirrhosis - such as retraction of the right liver lobe - which reduce the ability to explore the liver via intercostal scans. The ability to detect the emergence of a small HCC is highly dependent on the expertise of the operator performing the examination and the availability of state-of-the-art equipment. When these requirements are met, surveillance has proved effective in detecting HCC at an early stage [12-15]. The value of US surveillance performed in a primary care setting by operators who do not have specific skills is questionable. If the expertise is not available, the efficacy of surveillance will be lost. Upon detection of a suspicious nodule, the recommended policy is to evaluate the patient in referral centers with optimal human and technical resources [1].

Unfortunately, the use of contrast-enhanced US did not result in increased sensitivity US in the detection of small HCC tumors. As a general rule, contrastenhanced US techniques are subject to the same limitations as any other US mode: if the baseline scan is unsatisfactory, the contrast-enhanced US study will be unsatisfactory as well [6]. In addition, as described later, the greatest contrast between tumor and liver parenchyma is seen during the short time of the arterial phase. While multidetector CT and dynamic MR sequences can automatically image the entire liver parenchyma in a few seconds, a comprehensive manual scanning of the whole liver with US during the arterial phase is hardly attainable, even when performing repeated contrast injections. Thus, there is currently no indication to use microbubble contrast agents to increase the detection rate of HCC in patients undergoing US surveillance [6].

\section{Characterization of HCC}

Carcinogenesis is often a multi-step process in cirrhosis. This process includes progression from cirrhotic nodule, to macroregenerative nodule, to low-grade dysplastic nodule (DN), to high-grade DN, to frank HCC [16]. Progression along the multi-step pathway is characterized by cytological and architectural changes [17]. Unfortunately, these entities show variable and overlapping features at baseline US, making reliable differential diagnosis impossible. Small HCC may appear hyperechoic, hypoechoic, or isoechoic with respect to the surrounding parenchyma, and is usually undistinguishable from macroregenerative nodule or DN. In addition, small hyperechoic HCC may be undistinguishable from hemangioma [18].

One of the key pathologic factors for differential diagnosis between HCC and non-malignant hepatocellular 

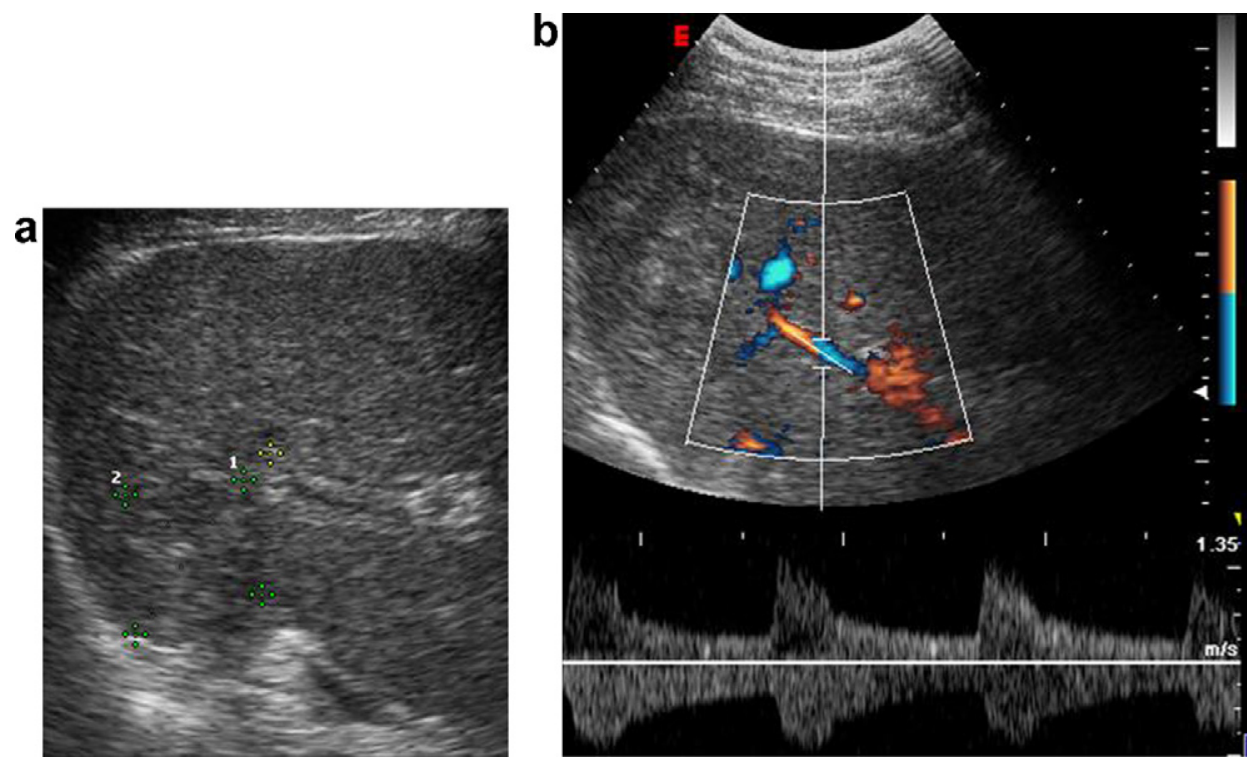

Fig. 2. Doppler US study of HCC. Baseline US scan (a) shows the tumor as hypoechoic nodule with infiltrative pattern (calipers). At Doppler US (b), flow in the segmental portal vein branch is reverted and shows a pulsatile pattern at spectral analysis, indicating the presence of arterio-portal fistula. Note that the peak of portal flow (shown below the zero line in the spectral tracing) is slightly delayed in comparison to that of the afferent hepatic arterial branch (above the zero line in the spectral tracing).

lesions that is reflected in imaging appearances is the vascular supply to the nodule. The progression from macroregenerative nodule, to low-grade $\mathrm{DN}$, to high-grade DN, to frank HCC, is characterized by loss of visualization of portal tracts and development of new arterial vessels, termed non-triadal arteries, which becomes the dominant blood supply in overt HCC lesions [19-21]. This arterial neoangiogenesis is the landmark of HCC and is the key for imaging diagnosis [22,23].

Doppler US techniques have long been used in attempts to evaluate tumor vascularity in $\mathrm{HCC}[24$ 27]. At color or power Doppler US, large HCC is usually displayed as a vascular-rich lesion containing intratumoral flow signals with an arterial Doppler spectrum. A basket pattern, which is a fine blood-flow network surrounding the nodule, and tumor vessels flowing into the lesion and branching within it are typically observed. Doppler interrogation shows a pulsatile Doppler waveform with high frequency shifts $(>1 \mathrm{kHz})$ and abnormally elevated resistive index $(>0.71)[25,27]$. Creation of arterio-portal fistula can be occasionally demonstrated (Fig. 2). In contrast, macroregenerative nodule and $\mathrm{DN}$ either do not have any detectable intratumoral vascularity or show arterial vessels with low frequency shifts and normal resistive index [28]. However, in small HCC tumors, the sensitivity of Doppler US in showing arterial neovascularity is low, and abnormal flow can be demonstrated in less than $50 \%$ of the lesions [24,28]. In addition, the technique is quite cumbersome and the positive predictive value is not high [29].

Several reports have shown that contrast-enhanced US is a tool to show arterial neoangiogenesis in HCC [30-33]. HCC typically shows strong intratumoral enhancement in the arterial phase, while macroregenerative nodule and DN usually do not show any early contrast uptake, and resemble the enhancement pattern of liver parenchyma. The ability of contrast-enhanced US to show arterial hypervascularization appears to approach that of optimized multidetector CT or dynamic MR imaging protocols, provided that the nodule can be clearly identified on baseline scans (Table 2).

Table 2

Studies comparing contrast-enhanced US with multidetector CT or dynamic MR imaging in the ability to detect arterial hypervascularization in nodular lesions in cirrhosis

\begin{tabular}{lllll}
\hline Author & No. lesions & Lesion size $(\mathrm{cm})$ & \multicolumn{2}{l}{ Detection rate } \\
\cline { 3 - 5 } & & & Contrast US (\%) & CT/MRI (\%) \\
\hline Gaiani et al. [34] & 103 & $2.8 \pm 1.3$ & 91 & CT, 100 \\
Bolondi et al. [35] & 41 & $1-2$ & 61 & CT, 49 \\
& 31 & $2-3$ & 97 & CT, 87 \\
Forner et al. [36] & 60 & $0.5-2$ & 78 & MRI, 85 \\
\hline
\end{tabular}

\footnotetext{
${ }^{a}$ This series included only tumors showing arterial hypervascularity in multidetector at CT.
} 
In one study, in which only HCC tumors showing arterial hypervascularity at CT were included, the sensitivity of contrast-enhanced US in the detection of arterial hyperenhancement was $91 \%$ [34]. In two comparative analyses including consecutive patients with small nodules in cirrhosis detected during surveillance, contrastenhanced US was superior to multidetector CT and slightly inferior to dynamic MR imaging in showing the presence of arterial hypervascularity (Table 2) $[35,36]$.

Unfortunately, the sole detection of arterial hypervascularity in a small nodular lesion emerged in a cirrhotic liver - although suspicious for HCC - may not be considered as a conclusive finding. It is well established that non-malignant hepatocellular lesions especially high-grade DN - may show arterial hypervascularization on imaging [37,38]. Small, high-flow hemangioma may also appear as a hyperenhancing nodule [39]. A recent investigation has shown that in the setting of cirrhotic patients undergoing surveillance, the sole imaging finding of arterial hypervascularization in small solitary nodules of $2 \mathrm{~cm}$ or less has a specificity of $86 \%$ and a positive predictive value of $92 \%$ for the diagnosis of HCC [36]. To increase the specificity of imaging diagnosis, it is mandatory to evaluate contrast wash-out during the portal venous and the late phase, a

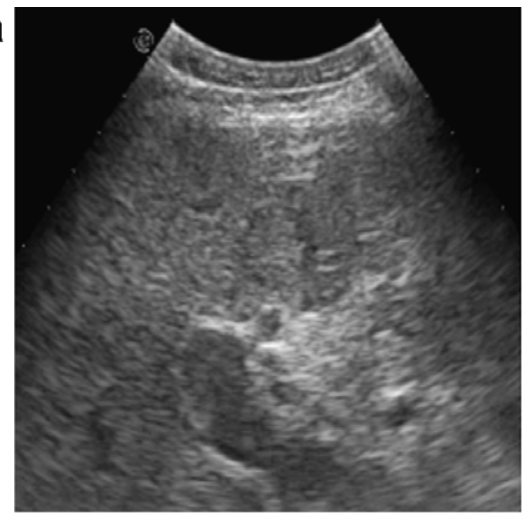

c

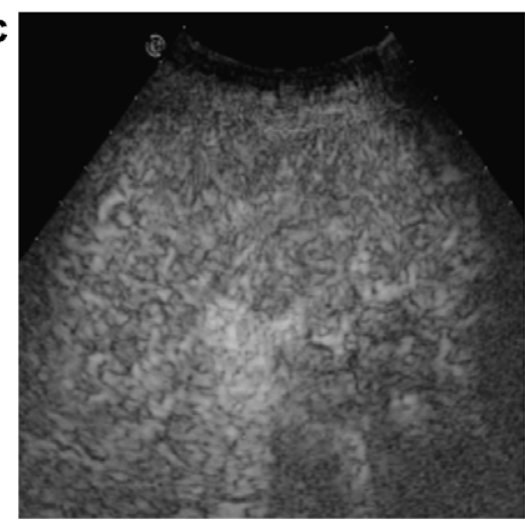

b

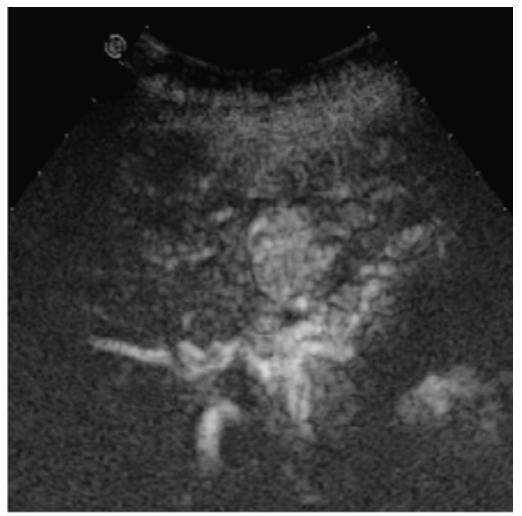

d

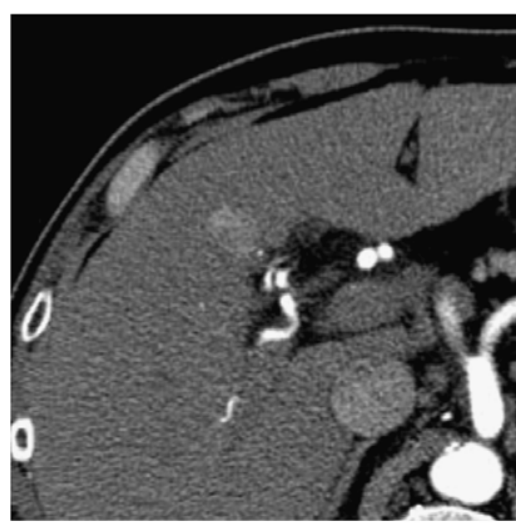

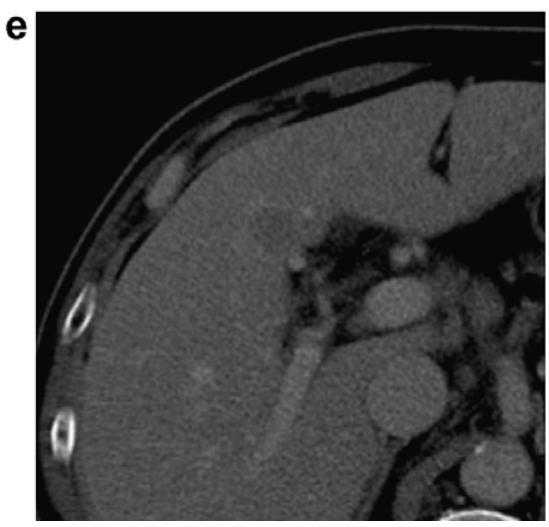

Fig. 3. Non-invasive diagnosis of very-early HCC in cirrhosis. Baseline US shows $1.5-\mathrm{cm}$ isoechoic nodule with hypoechoic halo (a). Contrast-enhanced US shows clear-cut enhancement in the arterial phase (b) with rapid wash-out in the portal venous phase (c). The same findings are observed on multidetector CT scans obtained in the arterial phase (d) and the portal venous phase (e). 
Table 3

Studies comparing contrast-enhanced US with multidetector CT or dynamic MR imaging in the diagnosis of HCC in nodules $2 \mathrm{~cm}$ or smaller detected during US surveillance

\begin{tabular}{|c|c|c|c|c|c|}
\hline Author & No. patients & No. lesions & Lesion size $(\mathrm{cm})$ & Sensitivity $(\%)$ & Specificity $(\%)$ \\
\hline Forner et al. [36] & 60 & $60^{\mathrm{a}}$ & $0.5-2$ & & \\
\hline Contrast US & & & & 52 & 93 \\
\hline Dynamic MRI & & & & 62 & 97 \\
\hline Dai et al. [40] & 72 & 103 & $1-2$ & & \\
\hline Contrast US & & & & 91 & 87 \\
\hline Multidetector CT & & & & 80 & 98 \\
\hline
\end{tabular}

Note. Figures refer to detection of typical enhancement pattern of HCC, i.e., arterial hypervascularization with venous wash-out.

${ }^{a}$ This series included only patients with solitary small tumors.

as recently recommended by the 2005 EASL conference on HCC and the AASLD practice guideline [2]. Contrary to non-malignant entities, HCC is characterized by rapid wash-out of the contrast agent, and usually appears hypoenhanced in the portal venous or the late phase [36-38] (Fig. 3).

If strict criteria - including hypervascularization in the arterial phase with wash-out in the portal venous or the late phase - are used to diagnose HCC, the very high specificity that can be obtained has the downside of a reduced sensitivity in the diagnosis of malignancy. In fact, the timing of contrast wash-out in HCC appears to be correlated with the degree of tumor differentiation. While moderately or poorly differentiated tumors have fast contrast wash-out and appear as defects in the portal venous or the late phase, well-differentiated tumors may wash out slowly and be isoenhanced with respect to the liver parenchyma in the portal venous or the late phase [4143]. Thus, diagnosis of small, well-differentiated tumors remains a challenge. Nevertheless, this is the case of any dynamic imaging technique, and the diagnostic accuracy of contrast-enhanced US for the diagnosis of HCC appears to be similar to that of multidetector CT or dynamic MR imaging (Table 3) $[36,40]$.

\section{Intrahepatic staging}

Accurate intrahepatic staging is essential for the proper clinical management of patients with HCC, particularly given the propensity of $\mathrm{HCC}$, even at early stages, to produce satellite lesions via the invasion of peripheral portal vein branches.

It is well established that US has limited sensitivity in the detection of tiny satellite lesions. When careful imaging-pathologic correlation on explanted livers was performed, the sensitivity of US was as low as $14 \%$ in the detection of lesions smaller than $2 \mathrm{~cm}$, and as low as $0 \%$ for cancerous foci smaller than $1 \mathrm{~cm}$ [44,45]. Although these data have been mostly collected in patients with advanced cirrhosis who underwent liver transplantation - and therefore may not be applicable to the general population of cirrhotic patients with HCC - the rate of underestimation of the extent of the disease with US is clearly unacceptable. Unfortunately, as discussed earlier, the use of contrast agents did not result in any significant improvement in the ability of US to detect small tumors [6]. The duration of the arterial phase - during which HCC tumors stand out against the faintly enhanced liver parenchyma - is far too short to allow a comprehensive manual scanning of the entire organ. In the portal venous and the late phase - contrary to hepatic metastases - the contrast between tumor and liver is usually low, preventing identification of small tumors not detected on baseline scans. Thus, even in the era of contrast-enhanced US, the use of either multidetector CT or dynamic MR imaging for intrahepatic staging of HCC is a mandatory step before therapeutic planning $[23,46]$.

In large HCC tumors, thrombosis of portal vein branches due to tumor invasion is commonly observed on imaging. However, in the setting of liver cirrhosis, portal vein thrombosis has a prevalence of about $5 \%$, even in the absence of HCC [47]. In patients with $\mathrm{HCC}$, distinction between malignant and non-malignant portal vein thrombosis is of paramount importance, as vascular invasion determines the shift from intermediate-stage to advanced-stage according to the Barcelona Clinic Liver Cancer (BCLC) staging system [48]. Recent observations have shown that contrast-enhanced US may be a tool for this purpose [49,50]. Unlike bland thrombosis, malignant thrombi show the typical features of HCC, and show rapid enhancement in the arterial phase due to the presence of hypervascularized tumor tissue (Fig. 4). In one study including 54 consecutive patients who had cirrhosis, biopsy-proven HCC, and thrombosis of the portal trunk or the main right or left branches, contrast-enhanced US showed absolute specificity and higher sensitivity than fine-needle biopsy in showing the malignant nature of the thrombus [49]. In another series of 34 patients listed for transplantation for HCC on cirrhosis, who also showed thrombosis of 


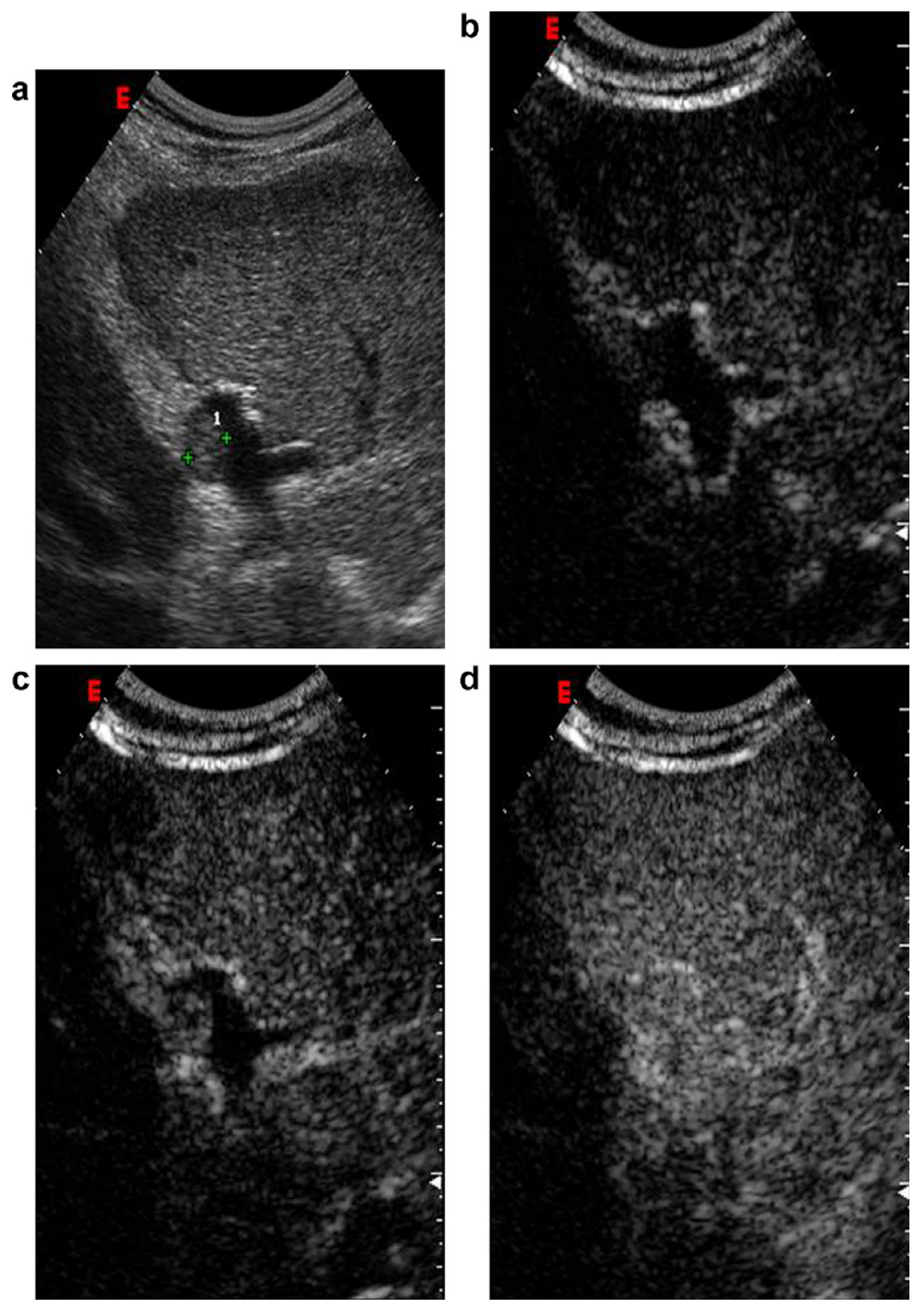

Fig. 4. Malignant portal vein thrombosis. Baseline US (a) shows tiny echoic thrombus (calipers) inside the left portal vein in patients with HCC. Signal from tissues is suppressed in the contrast-specific US image (b). Clear-cut contrast uptake of the thrombus is observed in the arterial phase of the contrastenhanced study, suggesting the malignant nature of the thrombus (c). In the portal phase, the portal lumen fills with contrast, and the thrombus is no longer recognizable (d).

the portal trunk or intrahepatic branches, contrastenhanced US was able to accurately exclude the malignant nature of the thrombus, as confirmed by pathologic analysis of the explanted organs [50].

\section{Diagnostic work-up}

The detection of a nodular lesion during US surveillance should always raise the suspicion of $\mathrm{HCC}$ $[1,2]$. However, pathologic studies have shown that a significant proportion of small nodules detected by
US in cirrhotic livers do not correspond to HCC, but rather to non-malignant hepatocellular nodules. Percutaneous US-guided biopsy could appear as the most straightforward approach to differentiate $\mathrm{HCC}$ from non-malignant hepatocellular lesions. Unfortunately, the biopsy of small nodular lesions in cirrhosis is not entirely reliable. In fact, needle placement may be difficult and a sampling error may occur. Moreover, it is very difficult to distinguish well-differentiated HCC from DN on small biopsy specimens, as there is not a clear-cut dividing line between dysplasia and well-differentiated tumor [51]. Therefore, a 
positive biopsy - as assessed by an expert pathologist - is helpful, but a negative biopsy can never be taken as a criterion to rule out malignancy [1,2]. In addition, biopsy is associated with a low but not negligible rate of complications, including tumor seeding along the needle track.

Both the 2005 EASL conference on HCC and the AASLD practice guideline have recommended to further investigate nodules detected during US surveillance with dynamic imaging techniques - including contrastenhanced US, multidetector CT, or dynamic MR imaging - to highlight the different vascular supply of HCC versus non-malignant entities [2]. However, the diagnostic protocol should be structured according to the actual risk of malignancy and the possibility to achieve a reliable diagnosis. Since the prevalence of HCC among US-detected nodules is strongly related to the size of the lesion, the diagnostic work-up depends on the size of the lesion [2]. Lesions smaller than $1 \mathrm{~cm}$ in diameter have a low likelihood of being HCC. However, such minute nodules may become malignant over time. Therefore, these lesions need to be followed up in order to detect growth suggestive of malignant transformation. A reasonable protocol is to repeat US every 3 months, until the lesion grows to more than $1 \mathrm{~cm}$, at which point additional diagnostic techniques are applied $[1,2]$. It has to be emphasized, however, that the absence of growth during the follow-up period does not rule out the malignant nature of the nodule because an early HCC may occasionally take more than one year to increase in size [1].

When the nodule exceeds $1 \mathrm{~cm}$ in size, the lesion is more likely to be HCC and diagnostic confirmation should be pursued. It is accepted that the diagnosis of HCC can be made without biopsy in a lesion larger than $1 \mathrm{~cm}$ that shows characteristic vascular features of HCC - i.e., arterial hypervascularization with washout in the portal venous or the late phase - even in patients with normal alpha-fetoprotein values [2]. While for lesions above $2 \mathrm{~cm}$ a single imaging technique showing the characteristic vascular profile of HCC mentioned above may confidently establish the diagnosis, in lesions ranging 1$2 \mathrm{~cm}$, AASLD guidelines recommend that typical imaging findings are confirmed by two coincident dynamic imaging modalities to allow a non-invasive diagnosis [2]. Such lesions ranging $1-2 \mathrm{~cm}$ are the true target of screening programs, as they identify the population of patients with very-early HCC tumors, who have the highest likelihood for cure with surgical resection or percutaneous ablation [52]. Specificity of imaging diagnosis is crucial to prevent therapeutic errors due to false-positive diagnoses of malignancy.

The combination of contrast-enhanced US and multidetector CT (or contrast-enhanced US and dynamic MR imaging) appears as the most rationale for noninvasive diagnosis of small tumors. This is clearly the most cost-effective combination, as the contrastenhanced US study can be performed immediately upon the detection of a focal lesion at baseline US, and only one additional examination (either CT or MR imaging) is needed as a confirmatory test. In addition, given the different pharmacokinetics of US contrast agents (blood-pool compounds) with respect to CT and MR imaging contrast agents (extracellular fluid space compounds), such a combination provides complementary information. In a prospective validation of AASLD criteria conducted in a series of consecutive patients with a solitary focal lesion smaller than $2 \mathrm{~cm}$ detected during US surveillance, the combined use of contrast-enhanced US and dynamic MR imaging achieved 100\% specificity for the diagnosis of HCC [36].

If the lesion does not show typical features of HCC, or the vascular profile is not coincidental between the imaging techniques, biopsy is recommended [2]. It is important to point out that the absence of arterial hypervascularization on imaging does not rule out HCC. It is well established that HCC tumors at a very-early stage may not exhibit the characteristic vascular features of overt HCC [35]. Delaying the diagnosis of HCC until imaging detection of arterial hypervascularization could reduce the chances of radical cure, since the incidence of microscopic vascular invasion and satellite nodules significantly increases when tumor exceeds $2 \mathrm{~cm}$ and develops imaging-detectable neoangiogenesis [17]. As a matter of fact, in the setting of cirrhotic patients in whom a solitary nodule smaller than $2 \mathrm{~cm}$ is detected during US surveillance, biopsy is still needed in about two-thirds of the cases, and - given the wellknown limitations of pathology reading - repeated biopsies are needed in as many as $30 \%$ of cases [36]. This is the area where advances in imaging techniques based on liver-specific contrast agents (including hepatocytetargeted agents and reticuloendothelial system-targeted agents) should be further investigated [53] and research on new diagnostic tools - based on immunostaining, gene expression assessment, or protein profiling - will have to focus [54].

\section{References}

[1] Bruix J, Sherman M, Llovet JM, Beaugrand M, Lencioni R, Burroughs AK, et al. EASL Panel of Experts on HCC. Clinical management of hepatocellular carcinoma. Conclusions of the Barcelona-2000 EASL conference. European Association for the Study of the Liver. J Hepatol 2001;35:421-430.

[2] Bruix J, Sherman M. Management of hepatocellular carcinoma. Hepatology 2005;42:1208-1236.

[3] Durand F, Regimbeau JM, Belghiti J, Sauvanet A, Vilgrain V, Terris B, et al. Assessment of the benefits and risks of percutaneous biopsy before surgical resection of hepatocellular carcinoma. J Hepatol 2001;35:254-258.

[4] Caturelli E, Solmi L, Anti M, Fusilli S, Roselli P, Andriulli A, et al. Ultrasound guided fine needle biopsy of early hepatocellular 
carcinoma complicating liver cirrhosis: a multicentre study. Gut 2004;53:1356-1362.

[5] Lencioni R, Cioni D, Bartolozzi C. Tissue harmonic and contrastspecific imaging: back to gray scale in ultrasound. Eur Radiol 2002;12:151-165.

[6] Albrecht T, Blomley M, Bolondi L, Claudon M, Correas JM, Cosgrove D, et al. EFSUMB Study Group. Guidelines for the use of contrast agents in ultrasound. Ultraschall Med 2004;25:249-256.

[7] Lencioni R, Cioni D, Crocetti L, Donati F, Franchini C, Giusti S, et al. Ultrasound imaging of focal liver lesions with a secondgeneration contrast agent. Acad Radiol 2002;9:S371-S374.

[8] Piscaglia F, Bolondi L; Italian Society for Ultrasound in Medicine and Biology (SIUMB) Study Group on Ultrasound Contrast Agents. The safety of Sonovue in abdominal applications: retrospective analysis of 23188 investigations. Ultrasound Med Biol 2006;32:1369-1375.

[9] Lencioni R, Cioni D, Crocetti L, Pina MCD, Bartolozzi C. Magnetic resonance imaging of liver tumors. J Hepatol 2004;40:162-171.

[10] Bolondi L. Screening for hepatocellular carcinoma in cirrhosis. J Hepatol 2003;39:1076-1084

[11] Colombo M. Screening. Hepatol Res 2007;37:S146-S151.

[12] Colombo M, de Franchis R, Del Ninno E, Sangiovanni A, De Fazio C, Tommasini M, et al. Hepatocellular carcinoma in Italian patients with cirrhosis. N Engl J Med 1991;325:675-680.

[13] Yuen MF, Cheng CC, Lauder IJ, Lam SK, Ooi CG, Lai CL. Early detection of hepatocellular carcinoma increases the chance of treatment: Hong Kong experience. Hepatology 2000;31:330-335.

[14] Bolondi L, Sofia S, Siringo S, Gaiani S, Casali A, Zironi G, et al. Surveillance programme of cirrhotic patients for early diagnosis and treatment of hepatocellular carcinoma: a cost effectiveness analysis. Gut 2001;48:251-259.

[15] Trevisani F, De Notariis S, Rapaccini G, Farinati F, Benvegnù L, Zoli M, et al. Semiannual and annual surveillance of cirrhotic patients for hepatocellular carcinoma: effects on cancer stage and patient survival (Italian experience). Am J Gastroenterol 2002;97:734-744.

[16] International Working Party. Terminology of nodular hepatocellular lesions. Hepatology 1995;22:983-993.

[17] Kojiro M, Roskams T. Early hepatocellular carcinoma and dysplastic nodules. Semin Liver Dis 2005;25:133-142.

[18] Caturelli E, Pompili M, Bartolucci F, Siena DA, Sperandeo M, Andriulli A, et al. Hemangioma-like lesions in chronic liver disease: diagnostic evaluation in patients. Radiology 2001;220:337-342.

[19] Ueda K, Terada T, Nakanuma Y, Matsui O. Vascular supply in adenomatous hyperplasia of the liver and hepatocellular carcinoma: a morphometric study. Hum Pathol 1992;23:619-626.

[20] Kimura H, Nakajima T, Kagawa K, Deguchi T, Kakusui M, Katagishi T, et al. Angiogenesis in hepatocellular carcinoma as evaluated by CD34 immunohistochemistry. Liver 1998;18:14-19.

[21] Roncalli M, Roz E, Coggi G, Di Rocco MG, Bossi P, Minola E, et al. The vascular profile of regenerative and dysplastic nodules of the cirrhotic liver: implications for diagnosis and classification. Hepatology 1999;30:1174-1178.

[22] Matsui O, Kadoya M, Kameyama T, Yoshikawa J, Takashima T, Nakanuma Y, et al. Benign and malignant nodules in cirrhotic livers: distinction based on blood supply. Radiology 1991;178:493-497.

[23] Lencioni R, Cioni D, Pina CD, Crocetti L, Bartolozzi C. Imaging diagnosis. Semin Liver Dis 2005;25:162-170.

[24] Lencioni R, Pinto F, Armillotta N, Bartolozzi C. Assessment of tumor vascularity in hepatocellular carcinoma: comparison of power Doppler US and color Doppler US. Radiology 1996;201:353-358.
[25] Gaiani S, Casali A, Serra C, Piscaglia F, Granmantieri L, Volpe $\mathrm{L}$, et al. Assessment of vascular patterns of small liver mass lesions: value and limitation of the different Doppler ultrasound modalities. Am J Gastroenterol 2000;95:3537-3546.

[26] Gaiani S, Volpe L, Piscaglia F, Bolondi L. Vascularity of liver tumours and recent advances in Doppler ultrasound. J Hepatol 2001;34:474-482.

[27] Fracanzani AL, Burdick L, Borzio M, Roncalli M, Monelli N, Borio F, et al. Contrast-enhanced Doppler ultrasonography in the diagnosis of hepatocellular carcinoma and premalignant lesions in patients with cirrhosis. Hepatology 2001;34:1109-1112.

[28] Lencioni R, Mascalchi M, Caramella D, Bartolozzi C. Small hepatocellular carcinoma: differentiation from adenomatous hyperplasia with color Doppler US and dynamic Gd-DTPAenhanced MR imaging. Abdom Imaging 1996;21:41-48.

[29] Teefey SA, Hildeboldt CC, Dehdashti F, Siegel BA, Peters MG, Heiken JP, et al. Detection of primary hepatic malignancy in liver transplant candidates: prospective comparison of CT, MR imaging, US, and PET. Radiology 2003;226:533-542.

[30] Isozaki T, Numata K, Kiba T, Hara K, Morimoto M, Sakaguchi $\mathrm{T}$, et al. Differential diagnosis of hepatic tumors by using contrast enhancement patterns at US. Radiology 2003;229:798-805.

[31] Quaia E, Calliada F, Bertolotto M, Rossi S, Garioni L, Rosa L, et al. Characterization of focal liver lesions with contrast-specific US modes and a sulfur hexafluoride-filled microbubble contrast agent: diagnostic performance and confidence. Radiology 2004;232:420-430.

[32] Chen MH, Dai Y, Yan K, Fan ZH, Yin SS, Yang W, et al. The role of contrast-enhanced ultrasound on the diagnosis of small hepatocellular carcinoma $(\angle /=3 \mathrm{~cm})$ in patients with cirrhosis. Hepatol Res 2006;35:281-288.

[33] Xu HX, Liu GJ, Lu MD, Xie XY, Xu ZF, Zheng YL, et al. Characterization of focal liver lesions using contrast-enhanced sonography with a low mechanical index mode and a sulfur hexafluoride-filled microbubble contrast agent. J Clin Ultrasound 2006;34:261-272.

[34] Gaiani S, Celli N, Piscaglia F, Cecilioni L, Losinno F, Giangregorio F, et al. Usefulness of contrast-enhanced perfusional sonography in the assessment of hepatocellular carcinoma hypervascular at spiral computed tomography. J Hepatol 2004;41:421-426.

[35] Bolondi L, Gaiani S, Celli N, Golfieri R, Grigioni WF, Leoni S, et al. Characterization of small nodules in cirrhosis by assessment of vascularity: the problem of hypovascular hepatocellular carcinoma. Hepatology 2005;42:27-34.

[36] Forner A, Vilana R, Ayuso C, Bianchi L, Solé M, Ayuso JR, et al. Diagnosis of hepatic nodules $20 \mathrm{~mm}$ or smaller in cirrhosis: Prospective validation of the noninvasive diagnostic criteria for hepatocellular carcinoma. Hepatology 2008;47:97-104.

[37] Valls C, Cos M, Figueras J, Andía E, Ramos E, Sánchez A, et al. Pretransplantation diagnosis and staging of hepatocellular carcinoma in patients with cirrhosis: value of dual-phase helical CT. AJR Am J Roentgenol 2004;182:1011-1017.

[38] Marrero JA, Hussain HK, Nghiem HV, Umar R, Fontana RJ, Lok AS. Improving the prediction of hepatocellular carcinoma in cirrhotic patients with an arterially-enhancing liver mass. Liver Transpl 2005;11:281-289.

[39] Kim T, Federle MP, Baron RL, Peterson MS, Kawamori Y. Discrimination of small hepatic hemangiomas from hypervascular malignant tumors smaller than $3 \mathrm{~cm}$ with three-phase helical CT. Radiology 2001;219:699-706.

[40] Dai Y, Chen MH, Fan ZH, Yan K, Yin SS, Zhang XP. Diagnosis of small hepatic nodules detected by surveillance ultrasound in patients with cirrhosis: comparison between contrast-enhanced ultrasound and contrast-enhanced helical computed tomography. Hepatol Res 2008;38:281-290. 
[41] Nicolau C, Catala V, Vilana R, Gilabert R, Bianchi L, Solé M, et al. Evaluation of hepatocellular carcinoma using SonoVue, a second generation ultrasound contrast agent: correlation with cellular differentiation. Eur Radiol 2004;14:1092-1099.

[42] Liu GJ, Xu HX, Lu MD, Xie XY, Xu ZF, Zheng YL, et al. Correlation between enhancement pattern of hepatocellular carcinoma on real-time contrast-enhanced ultrasound and tumour cellular differentiation on histopathology. $\mathrm{Br} \quad \mathrm{J}$ Radiol 2007;80:321-330.

[43] Jang HJ, Kim TK, Burns P, Wilson SR. Enhancement patterns of hepatocellular carcinoma at contrast-enhanced US: comparison with histologic differentiation. Radiology 2007;244:898-906.

[44] Bennett GL, Krinsky GA, Abitbol RJ, Kim SY, Theise ND, Teperman LW. Sonographic detection of hepatocellular carcinoma and dysplastic nodules in cirrhosis: correlation of pretransplantation sonography and liver explant pathology in 200 patients. AJR Am J Roentgenol 2002;179:75-80.

[45] Liu WC, Lim JH, Park CK, Kim MJ, Kim SH, Lee SJ, et al. Poor sensitivity of sonography in detection of hepatocellular carcinoma in advanced liver cirrhosis: accuracy of pretransplantation sonography in 118 patients. Eur Radiol 2003;13:1693-1698.

[46] Burrel M, Llovet JM, Ayuso C, Iglesias C, Sala M, Miguel R, et al. MRI angiography is superior to helical CT for detection of HCC prior to liver transplantation: an explant correlation. Hepatology 2003;38:1034-1042.

[47] Gaiani S, Bolondi L, Li Bassi S, Zironi G, Siringo S, Barbara L. Prevalence of spontaneous hepatofugal portal flow in liver cirrhosis. Clinical and endoscopic correlation in 228 patients. Gastroenterology 1991;100:160-167.

[48] Llovet JM, Bru C, Bruix J. Prognosis of hepatocellular carcinoma: the BCLC staging classification. Semin Liver Dis 1999;19: $329-338$.

[49] Tarantino L, Francica G, Sordelli I, Esposito F, Giorgio A, Sorrentino $\mathrm{P}$, et al. Diagnosis of benign and malignant portal vein thrombosis in cirrhotic patients with hepatocellular carcinoma: color Doppler US, contrast-enhanced US, and fine-needle biopsy. Abdom Imaging 2006;31:537-544.

[50] Piscaglia F, Gianstefani A, Ravaioli M, Sagrini E, Lodato F, Morelli $\mathrm{C}$, et al. Reliability of imaging techniques in assessing portal venous thrombosis in patients with hepatocellular carcinoma candidates for liver transplantation. Ultraschall Med 2007;28:S35.

[51] Kojiro M. Diagnostic discrepancy of early hepatocellular carcinoma between Japan and West. Hepatol Res 2007;37:S121-S124.

[52] Llovet JM, Burroughs A, Bruix J. Hepatocellular carcinoma. Lancet 2003;362:1907-1917.

[53] Bartolozzi C, Crocetti L, Lencioni R, Cioni D, Pina CD, Campani D. Biliary and reticuloendothelial impairment in hepatocarcinogenesis: the diagnostic role of tissue-specific MR contrast media. Eur Radiol 2007;17:2519-2530.

[54] Llovet JM, Chen Y, Wurmbach E, Roayaie S, Fiel MI, Schwartz $\mathrm{M}$, et al. A molecular signature to discriminate dysplastic nodules from early hepatocellular carcinoma in HCV cirrhosis. Gastroenterology 2006;131:1758-1767. 